\title{
Optical and Acoustic Study of Nucleation and Growth of Bubbles at a Liquid-Solid Interface Induced by Nanosecond-Pulsed-Laser Heating
}

\author{
O. Yavas ${ }^{1}$, P. Leiderer ${ }^{1}$, H. K. Park ${ }^{2}$, C. P. Grigoropoulos ${ }^{2}$, C. C. Poon ${ }^{3}$, W. P. Leung ${ }^{3}$, N. Do ${ }^{3}$, A. C. Tam ${ }^{3}$ \\ 1 Department of Physics, University of Konstanz, D-78434 Konstanz, Germany (Fax: +49-7531/88-3091) \\ 2 Department of Mechanical Engineering, University of California, Berkeley, CA 94720. USA (Fax: +1-510/642-6163) \\ 3 IBM Almaden Research Center, 650 Harry Road, San Jose, CA 95120-6099, USA (Fax: +1-408/927-2100)
}

Received 17 June 1993/Accepted 29 October 1993

\begin{abstract}
The dynamics of liquid-vapor phase-change in the nanosecond time-scale induced by pulsed-laser heating of a liquid on a solid sample is studied by means of optical reflectance and scattering measurements, and the piezoelectric detection technique. The liquids studied include water, ethanol, methanol, IsoproPropyl Alcohol (IPA), and mixtures of water and IPA. The threshold fluence for nucleation is determined with high accuracy using the optical and acoustic signals. Heat diffusion calculations performed for the threshold fluences indicate that the liquids are sufficiently superheated before nucleation sets on. The transient optical reflectance signal is analyzed by an effective-medium theory to provide bubble-growth kinetics, so that the bubble-growth velocity for the test liquids could be estimated. In addition, it is observed that, following the thermally induced nucleation, repetitive acoustic cavitation at the surface of the solid sample occurs, with a time interval related to the speed of sound in the liquid.
\end{abstract}

PACS: $64.70 . \mathrm{Fx}$, 79.20.Ds

The science of the metastability behavior of superheated pure or mixed liquids is well established only in the nearsteady-state regime with a time scale of microseconds or longer [1-4]. In the much faster regime with a time-scale of nanoseconds, superheated liquids may have different behavior, including the limit of the superheat, bubble nucleation and growth kinetics, and heterogeneous nucleation process at a surface. Such behaviors in the fast regime have not been investigated, because the traditional experimental techniques, such as short-pulsed electrical-current heating [1] and bubble column apparatus [5], lack speed and sensitivity. Recently, we have used pulsed-laser heating to produce faster superheating of liquids in the nanosecond-time-scale. In pulsed-laser heating the liquid can be heated directly if it is absorptive at the laser wavelength, as has been the approach in the heating of a liquid with the free surface in a container [6], or in the studies of explosive vaporization of aerosol droplets by $\mathrm{CO}_{2}$ lasers [7,8]. Alternatively, the liquid can be heated indirectly if it is transparent to the laser wavelength but is in contact with an opaque solid sample which absorbs the laser light. Recently, it was observed that this kind of liquid evaporation can be efficiently used to remove submicron-sized particles from solid surfaces $[9,10]$. Due to the practical significance of this "steam laser cleaning", many physical aspects such as nucleation dynamics, explosion thresholds for different liquids and substrates, and the pressures generated by the evaporation process need detailed investigation. Various methods including piezoelectric detection [11], optical transmission probe [12, 13], and probe-beam deflection [14] have been applied previously to the studies of these phenomena with some experimental restrictions.

A more general method for the study of nucleation and growth of bubbles at a liquid-solid interface is the optical reflectance probe, which can be applied to opaque solid samples of any thickness. Recently, we have reported for the first time that the threshold fluence for nucleation could accurately be determined from the optical reflectance transients, which could also be analyzed by an effectivemedium theory to provide bubble-growth dynamics [15]. Here, we present, in addition to the optical reflectance and scattering monitoring, the simultaneous application of the piezoelectric transducer measurements to the study of nucleation dynamics and explosive vaporization of various liquids on an opaque solid sample that is flash heated by a short laser pulse. In an analogy to the reflectance signal, it is also observed that the transient acoustic signal exhibits a threshold behavior which confirms bubble nucleation. Furthermore it is observed that the pressure wave generated by the liquid explosion causes repetitive cavitations at the surface of the solid sample.

\section{Experimental Arrangement and Procedure}

The experimental setup is shown in Fig. 1. The liquid is filled in a cuvette made of fused quartz. The dimensions of the cuvette are chosen so as to minimize possible lensing effects and free surface effects of the liquid. The solid sample is mounted on a massive aluminum block immersed 


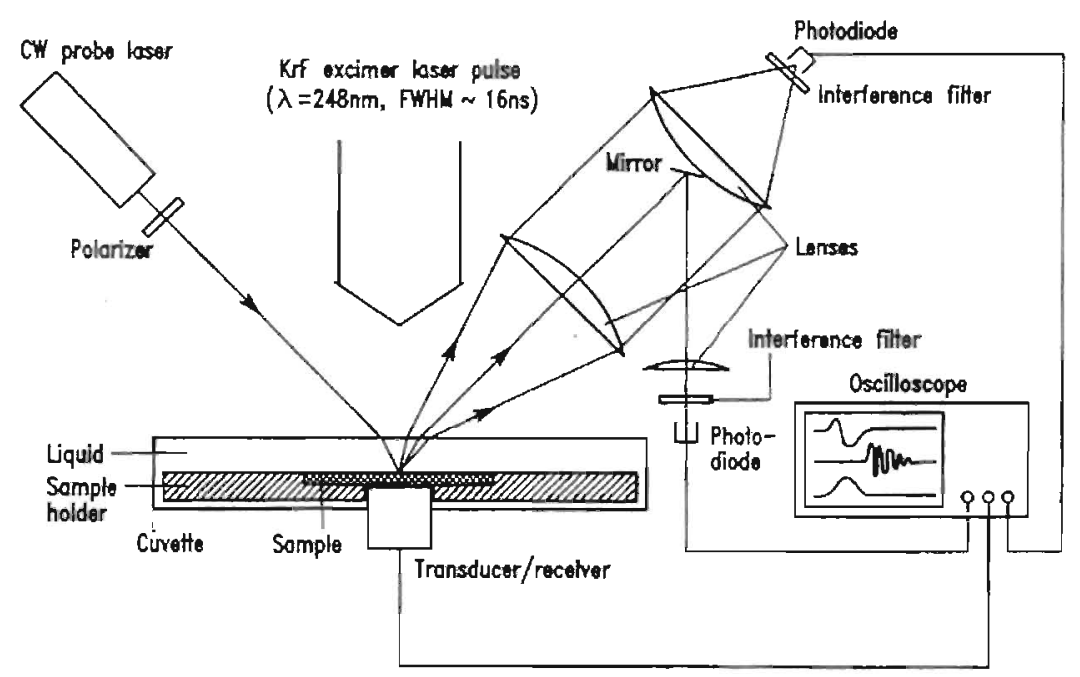

Fig. 1. Experimental serup for the measurement of optical reflectance and scattening, and acoustic resonance signal to probe the nucleation and growth of bubbles at a liquid-solid interface induced by puised $248 \mathrm{~nm} \mathrm{KrF}$-excimer-laser hearing entirely into the liquid. The $248 \mathrm{~nm} \mathrm{KrF}$-excimer laser pulse (FWHM 16ns) is directed at normal incidence onto the sample and irradiates a relatively large area $(1 \mathrm{~cm} \times 1 \mathrm{~cm}$ ). The probe beam is directed to the center of this area at an angle of incidence adjustable from $7^{\circ}$ to $46^{\circ}$. The specularly reflected and the scartered light, and the acoustic signal can be detected simultaneously by fast photodetectors (rise time $<1 \mathrm{~ns}$ ) and by a piezoelectric transducer (bandwidth $20 \mathrm{MHz}$ ). A thin liquid film ( $\sim 0.5 \mathrm{~mm}$ thick) couples the transducer acoustically to the sample. All the signals are monitored on a digital storage oscilloscope (Tektronix TDS540) triggered by the excimer-laser pulse. Several liquids including water, IPA, ethanol, methanol, and mixtures of water and IPA have been studied. The absorbing solid sample was at first a $0.2 \mu \mathrm{m}$ thick amorphous silicon film on a quartz substrate; the optical properties of amorphous silicon are temperature-dependent making the analysis of the data quite cumbersome. Therefore $0.2 \mu \mathrm{m}$ thick chromium films on a sapphire substrate ( $250 \mu \mathrm{m}$ thick) have been used for the present studies, because the optical properties of chromium are expected to be insensitive to the induced temperature changes. Sapphire was chosen as a substrate due to its high thermal conductivity causing a rapid cooling of the chromium film and limiting the time for superheating the liquid to tens of nanoseconds. By performing a careful position-sensing experiment using a photodetector of a large area ( $5 \mathrm{~mm} \times 5 \mathrm{~mm}$ ), a bicell, and a knife edge, we have verified that there is no measurable contribution to the reflectance signal due to any probe-beam deflection. Accordingly, any change on the reflectance signal can be ascribed to nucleation and growth of bubbles at the liquid-solid interface.

\section{Experimental Results and Discussion}

\subsection{Optical Reflectance Measurements}

The experiment is first conducted by irradiating the chromium sample in air using excimer-laser fluences up 10 $100 \mathrm{~mJ} / \mathrm{cm}^{2}$. No change in the reflectance signal is observed indicating that the optical properties of the chromium film are temperature-independent in the present temperature range. The computations based on the heat diffusion equation result in a peak temperature of $711 \mathrm{~K}$ for this highest applied fluence of $100 \mathrm{~mJ} / \mathrm{cm}^{2}$ (see Sect. 3). When the experiment is conducted with liquid in the cuvette, the reflectance signal shows distinct transients above a certain liquid-dependent threshold fluence. Figure 2 shows one set of the transient reflectance signals with increasing excimer-laser fluence for IPA and water, where the probe beam of a $752 \mathrm{~nm}$ diode laser is s-polarized and incident at an angle of $7^{\circ}$. Similar transients with minor differences are obtained for all the investigated liquids. The shape of the reflectance signal depends on the angle of incidence and the polarization of the probe beam. When the angle of incidence is small $\left(<40^{\circ}\right)$ the same shape for the transient reflectance signal is observed both for $s$ - and $p$-polarized probe beam: an abrupt reflectance increase followed by a more gradual dip. When the angle of incidence exceeds $40^{\circ}$, however, the leading positive part of

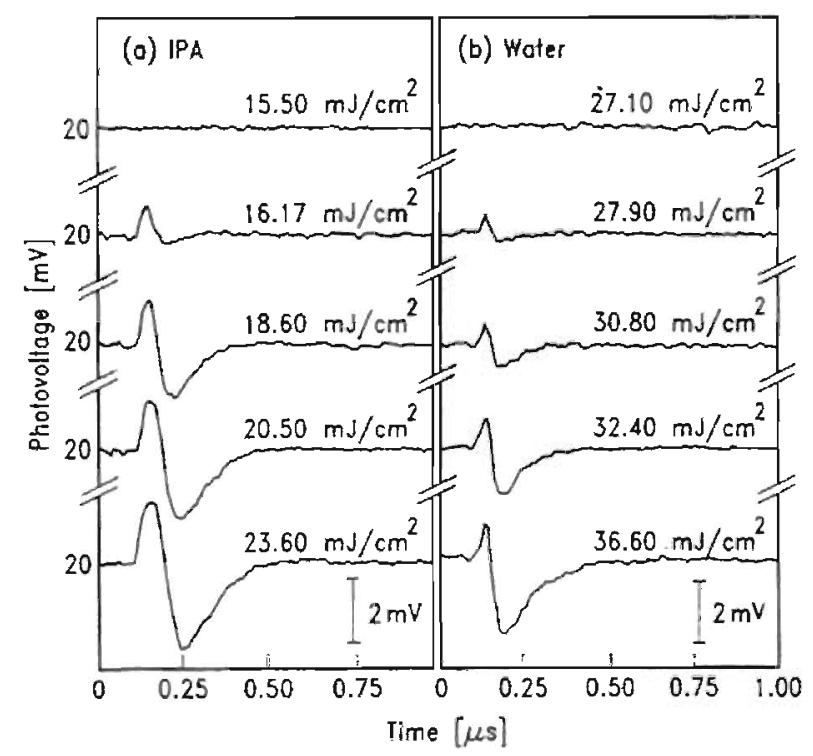

Fig. 2a, b. Transient reflectance signa] with increasing excimer laser fiuence for $0.2 \mu \mathrm{m}$ chromium film, $\lambda_{\text {probe }}=752 \mathrm{~nm}$. $s$-polarized, $\theta=7^{\circ}$. a IPA, b water 


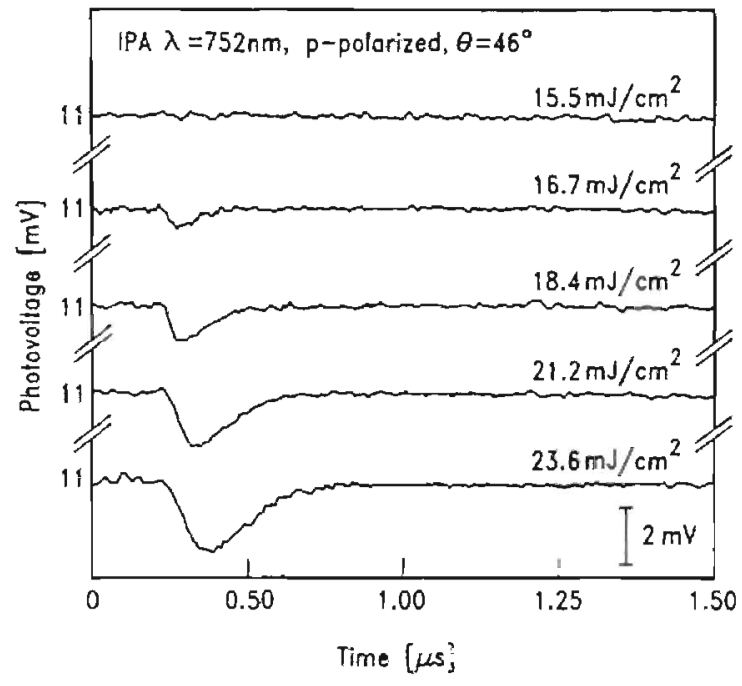

Fig. 3. Transient reflectance signal with increasing excimer-laser fluence for IPA in contacl with a $0.2 \mu \mathrm{m}$ chromium film, $\lambda_{\text {probe }}=752 \mathrm{~nm}$, $p$-polarized, $\theta=46^{\circ}$

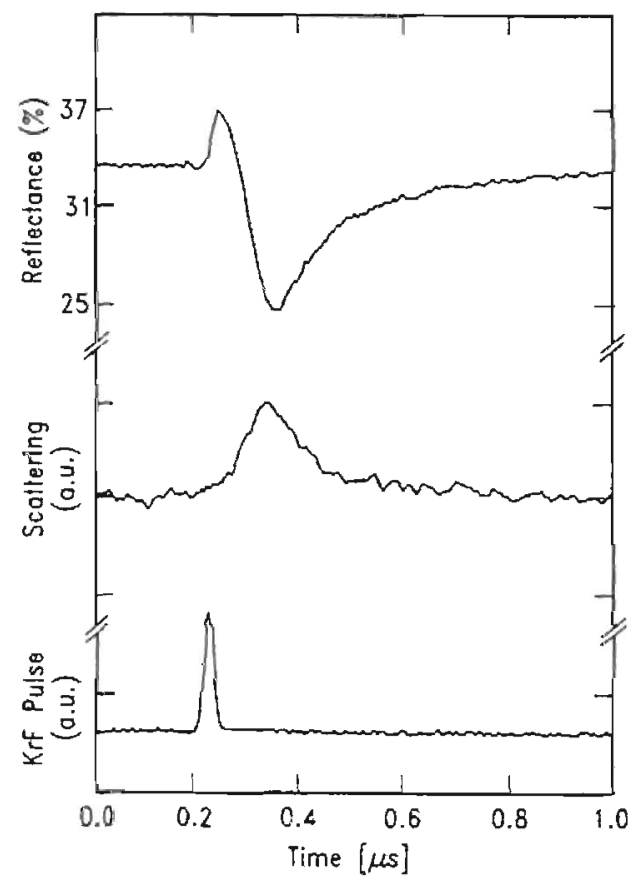

Fig. 4. Transient reflectance (top curve) and scattering (middle curve) signals for IPA measured simultaneously. $\lambda_{\text {probe }}=633 \mathrm{~nm}$, unpolarized, $\theta=20^{\circ}$. Lower curve represents the $\mathrm{KrF}$-excimer pulse $\left(F=32 \mathrm{~mJ} / \mathrm{cm}^{2}\right)$

the signal is only observable for the s-polarized probe beam. The result for the $p$-polarized probe beam incident at an angle of $46^{\circ}$ is shown in Fig. 3, for IPA as a test liquid. Note that the time for the signal to retum to the initial reflectance level before heating is comparable to that in Fig. 2, with the only difference that the initial peak is not observed. Previous studies using the optical transmission probe have already indicated that above a certain threshold fluence bubble nucleation at the liquid-solid interface occurs causing light scattering and a subsequent decrease of the transient transmission signal [12]. The same argument holds also for the reflectance signal decrease. This is well confirmed by the results of the simultaneous measurements of the specular reflectance and scattered light as shown in Fig. 4. The probe beam is unpolarized and the angle of incidence is $20^{\circ}$. The scattered light is collected with the help of two short focal length lenses with a collection angle of about $50^{\circ}$ from the forward direction (see Fig. 1). The increase of the scattering signal is in coincidence with the decrease of the specular reflectance signal. The initial increasing peak of the reflectance signal, on the other hand, seems at first sight to be inconsistent with the above argument. It could, however, be explained by means of the effective-medium theory by Maxwell-Gamett [16] which will be discussed in detail in the Sect. 2.4.

\subsection{Piezoelectric Transducer Measurements}

The change in the reflectance signal is accompanied by a sudden increase of the amplitude of the acoustic signal detected by the piezoelectric transducer simultaneously. Figure 5a shows a set of transient acoustic signals for a $0.2 \mu \mathrm{m}$ thick chromium film immersed in IPA. The corresponding optical reflectance signals obtained simultaneously are shown in Fig. 5b. Since the transducer is placed at the nonirradiated side of the sample, the acoustic wave generated at the liquid/Cr interface has to penetrare the chromium film, the sapphire substrate, and the liquid film in order to be detected. Due to the large acoustic impedance mismatch at the $\mathrm{Cr} /$ /iquid and sapphire/liquid interface (the acoustic impedance, which is given by $Z=\varrho c$, with $\varrho$ the density of the medium, and $c$ the speed of sound in it, is calculated to be $\sim 10^{7}, \sim 10^{7}$, and $\sim 10^{6} \mathrm{~kg} \mathrm{~m}^{-2} \mathrm{~s}^{-1}$ for chromium, sapphire, and the liquid, respectively) the acoustic wave is strongly reflected at these interfaces [17]. This causes a resonance in the sapphire substrate, which predominantly determines the shape of the transient acoustic signal since resonances in other media are considerably weaker. Thus, the observed frequency of the acoustic signal $(\sim 11 \mathrm{MHz})$ corresponds to the first fundamental natural frequency of the sapphire substrate [18]. This is calculated to be $11.2 \mathrm{MHz}$ according to the relation

$\nu_{\text {acou }}=\frac{c_{\text {sapp }}}{4 d_{\text {sapp }}}$,

where $c_{\text {sapp }}=11.2 \times 10^{3} \mathrm{~m} / \mathrm{s}$, is the velocity of sound in sapphire [19] and $d_{\text {sapp }}=250 \mu \mathrm{m}$. is the thickness of the sapphire substrate in the present studies. At low laser fluences the acoustic wave is generated thermo-elastically $[11,14]$ and its amplitude increases in direct relation to the induced temperature at the sample surface. When the laser fluence exceeds the nucleation threshold, however, the amplitude of the acoustic wave is increased by an additional amoun due to the explosive bubble growth and collapse. The generation of strong pressure due to bubble collapse, often causing undesired cavitation damage on technical equipment, such as propeller blades, pumps, and hydraulic machines, is known for many years [20] and can also be used to remove particulate contaminants from solid surfaces (ultrasonic and megasonic cleaning) [21]. 

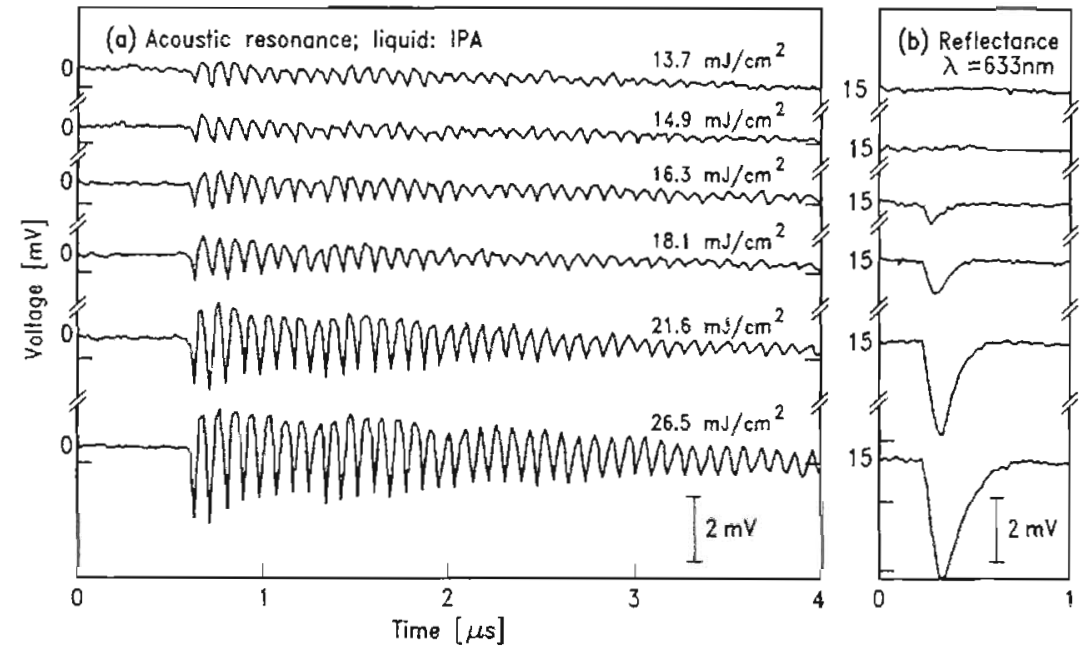

Fig. 5. a Transient acoustic signals for a $0.2 \mu \mathrm{m}$ thick chromium film immersed in IPA induced by $248 \mathrm{~nm} \mathrm{KrF}$-excimer-laser irradiation (FWHM $\sim 16 \mathrm{~ns}$ ) for various laser fluences. b The simultaneously observed optical reflectance signals, $\lambda_{\text {probe }}=633 \mathrm{~nm}$, p-polarized, $\theta=45^{\circ}$

\subsection{Correlation between the Optical Reflectance Transients and Bubble Growth}

The experimental results can be explained by the following physical picture: a uniform layer of bubbles is created at the surface of the chromium film when it is suddenly heated above the saturation temperature of the liquid. Due to the fast heating process, superheating above the boiling point is expected, until nucleation of bubbles sets in at a "cloud point", which marks the limit of supersaturation, as it is similarly observed during phase separation in a supersaturated fluid [22]. Initially, the radius of the bubbles scales with the surface roughness of the chromium film and is much smaller than the probe beam wavelength. At this stage, the bubble growth is inertia-controlled $[1,23]$ and the bubbles start to grow almost instantaneously without any significant time delay. An estimate of the time delay for the bubble growth by considering simple momentum balance between pressure force and inertia force [23], and by assuming that the liquid is superheated 20 degrees above its boiling point, results in a waiting time $<1 \mathrm{~ns}$, which is below the experimental resolution. In this initial stage of bubble growth, as long as the average bubble radius $R \ll \lambda_{\text {probe }} / 2 \pi n_{\text {liq }}$, where $n_{\text {liq }}$ is the refractive index of the test liquid, Rayleigh scattering dominates [24,25], and depending on the angle of incidence and polarization of the probe beam an increase or decrease of the reflectance can be observed. At later time when bubbles grow in size, $R \gg \lambda_{\text {probe }} / 2 \pi n_{\text {liq }}$, Mie scattering becomes important, resulting in a decrease of the specular reflectance. When the bubbles detach from the chromium surface or collapse, the reflectance signal returns to the initial level before heating.

\subsection{Modeling of Bubble Growth by an Effective-Medium Theory}

The initial stage of bubble growth can be modeled by Maxwell-Garnett's effective-medium theory [16], where three basic assumptions are needed: the bubbles are spherical, their average size is much smaller than the probebeam wavelength in liquid, and macroscopically they are distributed uniformly at the interface. With these assump- tions, the ensemble of small bubbles in a liquid is considered as a medium with an effective dielectric constant:

$\varepsilon_{\text {eff }}=\varepsilon_{1}\left(1-\frac{3 f\left(\varepsilon_{1}-\varepsilon_{v}\right)}{2 \varepsilon_{1}+\varepsilon_{\mathrm{v}}+f\left(\varepsilon_{1}-\varepsilon_{\mathrm{v}}\right)}\right)$

where $f$ is the fractional volume of bubbles, and $\varepsilon_{1}$ and $\varepsilon_{\mathrm{v}}$ are the dielectric constants for liquid and vapor, respectively. The effective dielectric constant simply equals to that of the liquid when $f=0$, i.e. when no bubble is present at the liquid/solid interface. It equals to that of the vapor, when $f=1$, i.e. when a vapor film is created at the liquid/solid interface. The system is considered as multilayered consisting of a bulk liquid ( $5 \mathrm{~mm}$ thick), a "foamy" liquid film with an effective dielectric constant $(0.05-5 \mu \mathrm{m}$ thick), a chromium
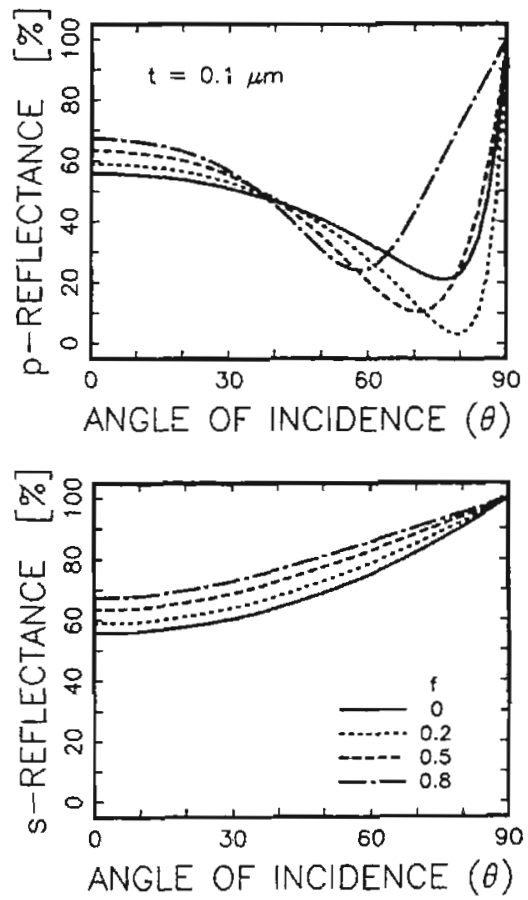

Fig. 6a, b. Computed refiectance based on the effective-medium theory (Maxwell-Gamett) for the system: foamy water film/Cr film/sapphire $(0.1 \mu \mathrm{m} / 0.2 \mu \mathrm{m} / 250 \mu \mathrm{m})$. $\mathrm{t}$ : thickness of the foamy film. f: fractional volume of the bubbles, $\lambda_{\text {probe }}=632.8 \mathrm{~nm}$, a $p$-polarized, $\mathrm{b}$ s-polarized 
film $(0.2 \mu \mathrm{m}$ thick), and a sapphire substrate $(250 \mu \mathrm{m}$ thick). Using thin-film optics we have calculated the reflectance for such a system for different angles of incidence, polarizations of the probe beam, and different thickness and bubble content of the foamy film. The optical constants for the bulk-Cr from the literature (at $632.8 \mathrm{~nm}, n=3.57$ and $k=4.36$ ) [39] have been used for the calculations. An example of the results for a $0.1 \mu \mathrm{m}$ thick foamy IPA film and for a probe beam of wavelength $632.8 \mathrm{~nm}$ is shown in Fig. 6 . The results are in qualitative agreement with the experimental observation: if the angle of incidence of the probe beam is small, i.e. $<40^{\circ}$, the reflectance signal increases with increasing fractional volume or initial bubble growth for both $s$ and $p$-polarization. If the angle of incidence exceeds $40^{\circ}$, however, the reflectance increases for $s$-polarization, whereas it decreases for $p$-polarization.

\subsection{Estimate of the Bubble-Growth Velocity}

It is well known that the transition from Rayleigh to Mie scattering occurs when the bubble radius is roughly in the order of $R_{\mathrm{ur}} \equiv \lambda_{\text {probe }} / 2 \pi n_{\text {lig }}[24,25]$. Accordingly, the average bubble-growth velocity can be estimared by assuming that the time of the initial peak in the reflectance signal (considering an $s$-polarized probe beam) corresponds to the time, $t_{\mathrm{t}}$, needed for a bubble to grow up to this specific transition radius, $R_{\mathrm{v}}$. In addition, it is recalled that the bubble radius increases linearly with time during the initial inertiacontrolled stage of bubble growth, and increases with the square root of time in the later heat-transfer-controlled stage [23]. We observe $t_{\mathrm{tr}} \sim 45 \mathrm{~ns}$ for IPA and $\sim 25 \mathrm{~ns}$ for water using a $752 \mathrm{~nm}$ probe laser (see Fig. 2). For this probe-beam wavelength, $R_{\mathrm{tr}}$ corresponds to $87 \mathrm{~nm}$ for IPA $\left(n_{\mathrm{liq}}=1.38\right)$ [26] and $90 \mathrm{~nm}$ for water $\left(n_{\text {liq }}=1.33\right)$ [26]. This results in an average bubble-growth velocity of $\sim 1.9 \mathrm{~m} / \mathrm{s}$ for IPA and $\sim 3.6 \mathrm{~m} / \mathrm{s}$ for water. The growth velocities estimated in the same way for the other investigated liquids are also found to be in this range, showing a trend in qualitative agreement with the predictions for the inertia-controlled bubble growth, which is theoretically described by the Rayleigh formulation [23]. The upper limit for it is given by [27]

$$
\left(\frac{d R}{d t}\right)_{\text {inerial }}=\left(\frac{2}{3} \frac{p_{v}\left(T_{\infty}\right)-p_{\infty}}{\varrho}\right)^{1 / 2}
$$

where $R$ is the bubble radius, $\varrho$ is the liquid density, $p_{\mathrm{v}}\left(T_{\infty}\right)$ is the vapor pressure at the superheated liquid temperature, and $p_{\infty}$ is the ambient liquid pressure. The peak temperature at the water/chromium interface induced by an excimerlaser pulse of $F=27.4 \mathrm{~mJ} / \mathrm{cm}^{2}$ (experimentally determined threshold fluence for nucleation of water), for instance, is calculated to be $426 \mathrm{~K}$ (see Sect. 3). However, there is a high temperature gradient in the water layer adjacent to the chromium surface and therefore its average temperature could be assumed to be $400 \mathrm{~K}$. The vapor pressure for water at this temperature is $247 \times 10^{3} \mathrm{~Pa}$ [23], resulting in an inertia-controlled bubble-growth velocity of $10 \mathrm{~m} / \mathrm{s}$. It is noted that the Rayleigh formulation for the inertia-controlled bubble growth, which is based on some idealizations [28], had been explored at longer, microsecond time scales [1]. The estimated growth velocities are, however, much smaller

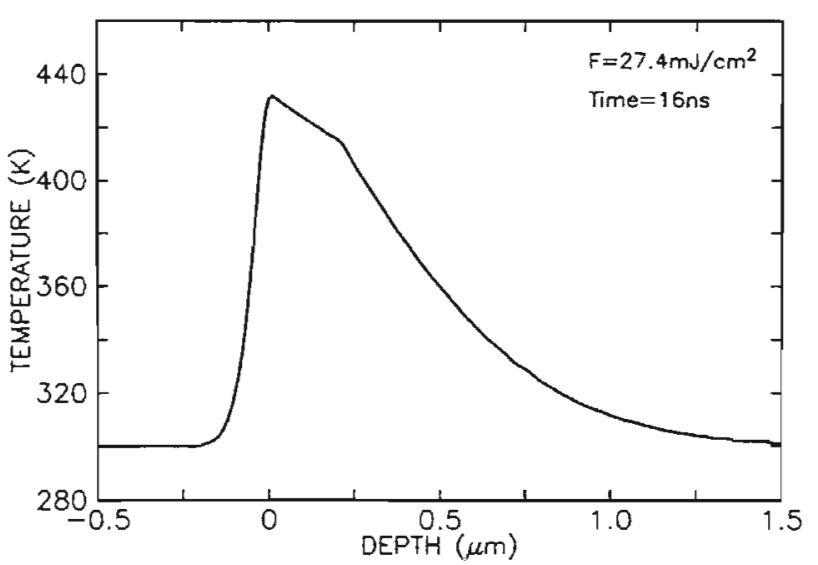

Fig. 7. Temperature profile in the system of water/chromium/sapphire $(1 \mu \mathrm{m} / 0.2 \mu \mathrm{m} / 5 \mu \mathrm{m}$ thick) calculated for a $248 \mathrm{~nm} \mathrm{KrF}$-excimer-laser of fuence $27.4 \mathrm{~mJ} / \mathrm{cm}^{2}$ using the numerical solution of the onedimensional heat diffusion equation. Thermophysical material properties given in Table 1 are used in the computation. The water/chromium interface is at depth $=0 \mu \mathrm{m}$

than the velocities observed in experiments for the inertiacontrolled stage of homogeneous bubble nucleation. For the homogeneous nucleation of butane, for example, a growth velocity of $14.3 \mathrm{~m} / \mathrm{s}$ was observed experimentally [29]. In the experiments presented here, bubbles form on the surface of the solid sample and are described by the heterogeneous nucleation theory, which predicts much less superheat for the onset of nucleation compared with the homogeneous nucleation theory [23]. In addition, the bubbles cannot grow beyond a certain radius which is limited by the thickness of the superheated liquid layer at the $\mathrm{Cr}$ surface. Thus, except for the very initial phase of growth, bubbles grow as the heat diffuses into the liquid, i.e. the speed of heat diffusion into the liquid limits the bubble-growth velocity. This is confirmed by the results of the numerical computations of the heat diffusion equation as shown in Fig. 7, where the temperature profile in the system of water/Cr/sapphire $(1 \mu \mathrm{m} / 0.2 \mu \mathrm{m} / 5 \mu \mathrm{m})$ is plotted at $16 \mathrm{~ns}$ after the onset of the excimer-laser irradiation of $F=27.4 \mathrm{~mJ} / \mathrm{cm}^{2}$, the nucleation threshold fluence for water. It is seen that only a thin layer of about one hundred nanometers of water is superheated at that moment, while the temperature at the water/chromium interface amounts to $426 \mathrm{~K}$.

\subsection{Wavelength Dependence of the Transient Reflectance Signal}

The above presented interpretation of the reflectance signal is confirned by probing the same location of the chromiumIPA interface with three lasers of different wavelengths $(\lambda=488,632.8$, and $752 \mathrm{~nm})$ under similar conditions, i.e. polarization and angle of incidence. The result shown in Fig. 8 is acquired with $s$-polarized probe beams incident at an angle of $10^{\circ}$, and for an excimer-laser fluence of $27 \mathrm{~mJ} / \mathrm{cm}^{2}$. A widening of the initial peak and a shift of its maximum to later times is observed with increasing probe beam wavelength as expected, since the bubble radius $R_{\mathrm{rr}} \equiv \lambda_{\text {probe }} / 2 \pi n_{\mathrm{liq}}$, at which the transition from Rayleigh to Mie scattering occurs, depends linearly on the probe beam wavelength. With the average growth velocity of bubbles 


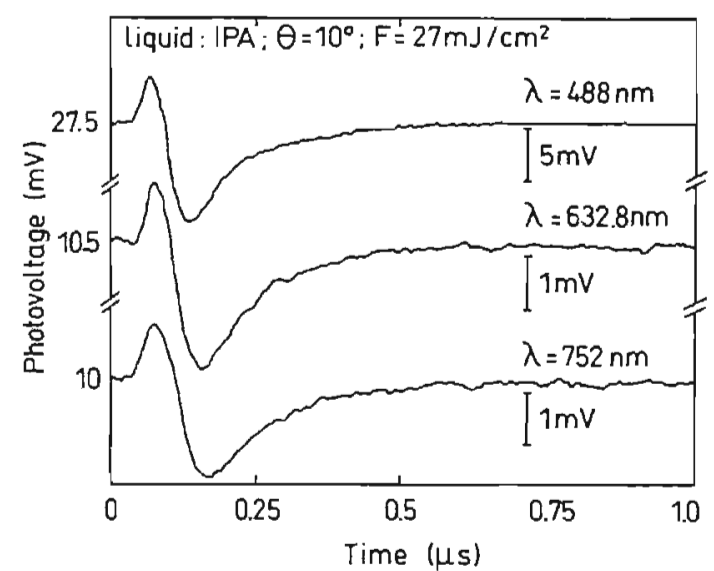

Fig. 8. Comparison of the reflectance transient for XPA at three different probe beam wavelengths. $\lambda_{\text {probe }}=488 \mathrm{~nm}(l o p), 632.8 \mathrm{~nm}$ (middle), and $752 \mathrm{~nm}$ (bottom), $\theta=10^{\circ}, s$-polarized

for IPA being $1.9 \mathrm{~m} / \mathrm{s}$, and the transition radii of $73 \mathrm{~nm}$ and $87 \mathrm{~nm}$ for the probe beam wavelengths of $632.8 \mathrm{~nm}$ and $752 \mathrm{~nm}$, a time delay of $7 \mathrm{~ns}$ is expected for the peak position of the two corresponding reflectance signals. The observed value $\sim 8 \mathrm{~ns}$ is in good agreement with this prediction and supports the proposed interpretation that the optical reflectance transient represents the bubble-growth dynamics.

\subsection{Determination of the Threshold Fluence for Nucleation}

The threshold fluence for the nucleation can be determined accurately when the amplitude of the reflectance drop is plotted versus the excimer laser fluence. The results for the test liquids are shown in Fig. 9. There is a liquiddependent threshold fluence above which the reflectance signal suddenly starts to drop. The threshold fiuences for the test liquids determined from this plot are listed in Table 1, together with boiling points and the calculated maximum temperatures at the liquid/chromium interface for the corresponding threshold fluences. The procedure of

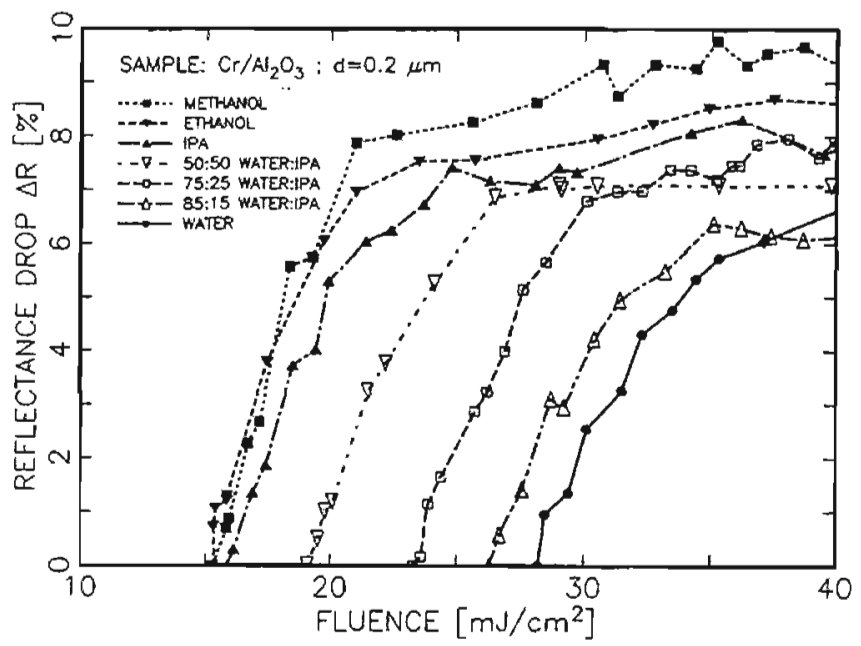

Fig. 9. Optical reflectance drop vs $248 \mathrm{~nm} \mathrm{KrF}$-excimer-laser fluence (FWHM $\sim 16 \mathrm{~ns}$ ) for different liquids in contact with a $0.2 \mu \mathrm{m}$ thick chromium film. The onset of reflectance drop provides the threshold fluence for nucleation. $\lambda_{\text {probe }}=632.8 \mathrm{~nm}$, p-polarized, $\theta=46^{\circ}$
Table 1. The experimentally determined $248 \mathrm{~nm}$ excimer-laser threshold fluences, $F_{\text {th }}$, for the onset of nucleation for different liquids, the boiling points, bp $[23,26,30]$, and the peak temperatures at the liquid/chromium interface, $T_{\mathrm{pk}}$, calculated for the corresponding threshold fluences by the numerical solution of the heat diffusion equation

\begin{tabular}{llll}
\hline Liquid & $F_{\text {th }}\left[\mathrm{mJ} / \mathrm{cm}^{2}\right]$ & bp $[\mathrm{K}]$ & $T_{p \mathrm{k}}[\mathrm{K}]$ \\
\hline Ethanol & 14.9 & 351.5 & 375 \\
Methanol & 15.4 & 337.9 & 377 \\
IPA & 15.7 & 355.6 & 379 \\
50:50 (Water: IPA) & 19.1 & 369.8 & 390 \\
$75: 25$ (Water: IPA) & 23.1 & 371.9 & 408 \\
85:15 (Water: IPA) & 25.9 & 372.5 & 420 \\
Water & 27.4 & 373.2 & 426 \\
\hline
\end{tabular}

the computation will be discussed in detail in Sect. 3. The nucleation threshold fluences for the test alcohols are found to be in a very narrow range around $15 \mathrm{~mJ} / \mathrm{cm}^{2}$. On the other hand, the threshold fluence for water $\left(27.4 \mathrm{~mJ} / \mathrm{cm}^{2}\right)$ is almost twice as high as those for alcohols, consistent with the higher boiling point of water. The sharp increase of the amplitude of the reflectance drop is followed by a much slower rate of change at about $10 \mathrm{~mJ} / \mathrm{cm}^{2}$ above the threshold fluence. This observation could be explained by a hypothesis that the bubbles can grow largely enough to agglomerate and build a continuous vapor layer at the solid surface when the superheat is sufficient. It is also possible that the bubble growth beyond a certain point impedes the activation of new nucleation sites. It is further observed that the threshold fluences for water/IPA mixtures do not depend linearly on the alcohol content (volume percent), which is consistent with the fact that the properties of liquid mixtures do not depend linearly on the volume or mole fraction of their constituents [30].

The threshold fluence for nucleation could also be determined from a plot of the acoustic resonance amplitude versus excimer laser fluence. Such a plot for water on the surface of a $0.2 \mu \mathrm{m}$ thick $\mathrm{Cr}$ film irradiated by $248 \mathrm{~nm} \mathrm{KrF}$-excimer laser is shown in Fig. 10. Below the threshold fluence for nucleation indicated by an arrow, the acoustic resonance amplitude increases linearly with the excimer-laser fluence since the laser energy is coupled thermo-elastically into the solid sample, i.e. the fast temperature rise at the surface of

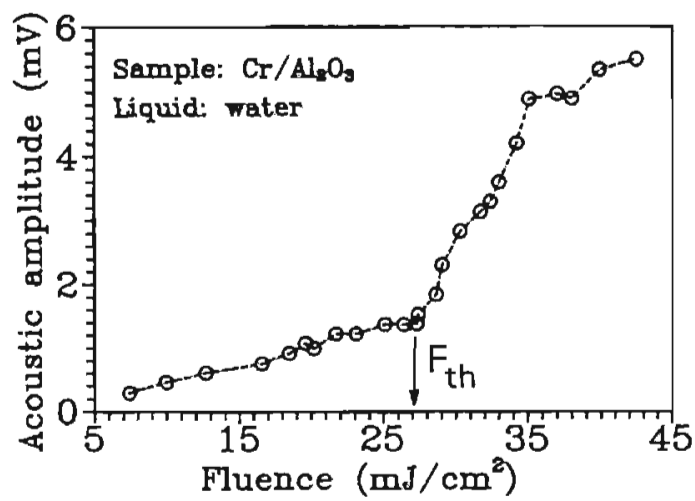

Fig. 10. Acoustic resonance amplitude versus $248 \mathrm{~nm}$ excimer-laser fluence ( $F W H M \sim 16 \mathrm{~ns}$ ) for water in contact with a $0.2 \mu \mathrm{m}$ thick chromium film. The line is a guide to the eye. The onset of the step indicated by the arrow marks the threshold fluence for nucleation 
the solid sample causes a sudden expansion of the irradiated area, resulting in acoustic wave generation $[11,14,31]$. In the case of a bare solid surface this linear dependence remains unchanged for excimer-laser fluences well above the threshold fluence for the onset of phase change in liquids. When liquid is present on the chromium surface, however, and the excimer-laser fluence exceeds the threshold for nucleation, the amplitude of the acoustic wave is additionally increased due to bubble growth and collapse, resulting in a sudden change of the slope of the curve, as seen in Fig. 10, which marks the threshold fluence for nucleation.

\subsection{Long-Time Scale Measurements and Repetitive Cavitation}

In a longer time scale, it is observed that the reflectance signal drops repeatedly in a certain time interval with decreasing amplitude, as shown in Fig. 11. The time interval between these "echo" signals depends on the distance between the chromium sample and the quartz window of the cuvette and on the speed of sound of the studied liquid. Thus, it corresponds to the time for the round-trip of the acoustic wave from the sample surface to the quartz/liquid interface and back. This kind of "echo" phenomena has been also observed earlier in the pulsed-laser-induced transient phase transformations at the $\mathrm{Si}-\mathrm{H}_{2} \mathrm{O}$ interface [32]. It is assumed that the first dip is due to bubble formation by superheating of the liquid, while the following echo signals are only due to acoustic cavitation. This assumption is justified by the results of the heat-diffusion calculations, which indicate that the temperature at the liquid/chromium interface is already decreased to room temperature at about $1 \mu \mathrm{s}$ after the excimer-laser irradiation, as shown in Fig. 12. The optical properties of water are nearly insensitive to pressure, for

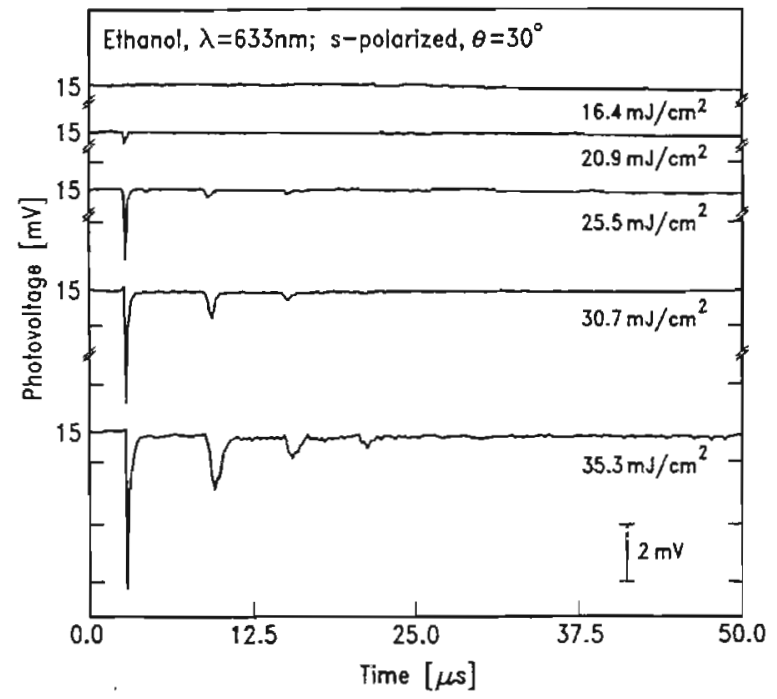

Fig. 11. Optical reflectance signal for ethanol in contact with a $0.2 \mu \mathrm{m}$ thick chromium film irradiated by $248 \mathrm{~nm} \mathrm{KuF-excimer} \mathrm{laser} \mathrm{(FWHM}$ $\sim 16 \mathrm{~ns}$ ) shows that cavitation takes place at the ethanol/chromium interface after each round-trip of the acoustic pulse in methanol between the chromium sample and the quartz window (distance samplequarz window $\approx 7 \mathrm{~mm}$ ). The decay in the signal amplitude is caused by attenuation due to diffraction, absorption and reflection losses

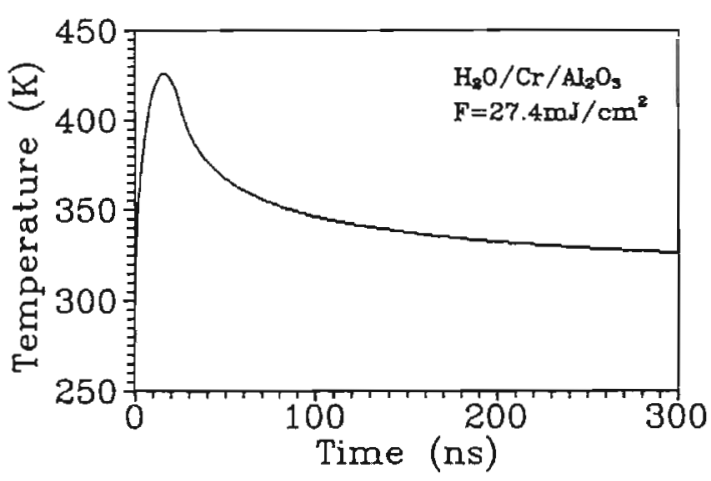

Fig. 12. The evolution of temperature at the water/chromium interface calculated for a $248 \mathrm{~nm} \mathrm{KrF}$-excimer-laser irradiation of $27.4 \mathrm{~mJ} / \mathrm{cm}^{2}$ using the numerical solution of the heat diffusion equation by the finite difference method. Thermophysical material properties given in Table 1 are used in the computation

instance, the refractive index of water at $\lambda=589 \mathrm{~nm}$ increases by only $0.12 \%$ as the pressure increases to 100 times the atmospheric pressure $[33,34]$. It is therefore concluded that the piezooptical effect or the lensing effect is negligible and the repetitive reflectance drops can be explained only by bubble formation. In addition, for all the echo signals, the acoustic signal is detected sooner by the piezoelectric transducer than the optical reflectance drop, confirming that the echoes of optical signals are caused by bubble formation due to acoustic cavitation. The bubbles giving rise to the initial reflectance drop could also be due partly to superheating and partly to cavitation. In this respect, acoustic cavitation needs to be studied in more detail using specially designed experiments. No reflectance echo is observed when the quartz window is probed. This result can be explained by the possible existence of residual ultramicroscopic bubbles at the irradiated $\mathrm{Cr}$ surface [35], which provide themselves as seeds for cavitation, consequently resulting in a lower cavitation threshold. The decrease of the reflectance drop in the successive echo signals is ascribed to the attenuation of the acoustic wave due to diffraction, absorption, and reflection losses. It should be possible to determine the cavitation thresholds for different liquids and solid samples by calibrating the amplitude of the transducer signal for absolute pressure measurements.

\section{Temperature Modeling}

The temperature at the liquid/chromium interface induced by the excimer-laser pulse is of fundamental interest for a better understanding of the nucleation process. It is expected that the liquid is superheated above the boiling point before nucleation sets on. The temperature profile penetration is of the order of $1 \mu \mathrm{m}$, whereas the laser beam spot area on the sample surface is measured to be about $1 \mathrm{~cm}^{2}$. Accordingly, it may be assumed that the heat transfer at the center of the irradiated area is essentially one-dimensional. Hence, we have calculated the temperature profile in the multilayered structures (liquid/chromium/sapphire) using the one-dimensional heat diffusion equation:

$o C_{p} \frac{\partial T}{\partial t}=\frac{\partial}{\partial z}\left(K \frac{\partial T}{\partial z}\right)+Q_{\mathrm{ab}}(z, t)$ 
Table 2. Thermophysical properies of chromium and water $[26,37,40.41]$ used for the heat-diffusion calcularions

\begin{tabular}{|c|c|c|}
\hline Material & Property & \\
\hline \multirow[t]{4}{*}{ Chromium } & $\varrho=7190\left[\mathrm{~kg} / \mathrm{m}^{3}\right]$ & \\
\hline & $K(T)=139.79-0.16475 T[W / \mathrm{mK}] \quad(273 \mathrm{~K} \leq T<311 \mathrm{~K})$ & \\
\hline & $=77.584+8.5188 \times 10^{-2} T^{-1}-1.8816 \times 10^{-4} T^{2}+9.0307 \times 10^{-8} T^{3}[\mathrm{~W} / \mathrm{mK}]$ & $(311 \mathrm{~K} \leq T \leq 1100 \mathrm{~K})$ \\
\hline & $C_{p}(t)=201.01+1.2517 T-1.6586 \times 10^{-3} T^{3}+8.0767 \times 10^{-7} T^{3}[\mathrm{~J} / \mathrm{kgK}]$ & \\
\hline \multirow[t]{3}{*}{ Water } & $a(T)=1000-7.792 \times 10^{-3}(T-273.2)^{1.8478}\left[\mathrm{~kg} / \mathrm{m}^{3}\right]$ & \\
\hline & $K(T)=-0.33 \div 4.92 \times 10^{-3} \mathrm{~T}-5.97 \times 10^{-6}[\mathrm{~W} / \mathrm{mK} \mathbf{j}$ & \\
\hline & $C_{p}(T)=4169.53+1.203\left(\frac{T-173.2}{100}\right)^{5.26}+467.1 T^{-0.036(T-273)}[\mathrm{J} / \mathrm{kgK}]$ & \\
\hline
\end{tabular}

where $z$ is the coordinate normal to the sample surface into the solid, $\varrho$ is the density, $T$ is the temperature, $C_{p}$ is the specific heat for constant pressure, and $K$ is the thermal conductivity. The temperature dependence of the material thermal properties are considered. The energy absorption, $Q_{\mathrm{ab}}(z, t)$, follows an exponential decay in the material:

$Q_{\mathrm{ab}}(z, t)=\left(1-\mathscr{B}_{\mathrm{exc}}\right) I(t) \alpha \mathrm{e}^{-\alpha z}$,

where $\mathscr{B}_{\text {exe }}$ is the reflectivity of the quartz window/liquid/. chromium sample for the excimer-laser light, $I$ is the incident laser-beam intensity, and $\alpha$ is the absorption coefficient. The heat diffusion equation is solved numerically by the finite-difference method $[13,36]$. The optical constants for the thin $\mathrm{Cr}$ film used for the computations were determined by ellipsometry measurements (at $\lambda=248 \mathrm{~nm}, n=1.2$ and $k=1.55$ ), which were found to be distinctly different from those for bulk- $\mathrm{Cr}$ ( $n=0.85$ and $k=2.01$ ) [39]. This is in agreement with the observarion that the optical properties of evaporated thin $\mathrm{Cr}$ films are, in general, different from those of bulk-Cr $[38,39]$. The temperature dependence of the thermophysical properties for bulk-chromium and water are listed in Table 2. It is noted that the thermophysical properties of thin $\mathrm{Cr}$ films may be different from those of bulk-Cr. In addition, a simple assumption is made that no phase change in the liquid occurs in the computation. The results of the computations indicate that the liquids are superheated tens of degrees above boiling points before the nucleation sets in. This can be seen in Fig. 12, showing the transient temperature profile at the water/chromium interface calculated for an excimer-laser fluence of $F=27.4 \mathrm{~mJ} / \mathrm{cm}^{2}$, which corresponds to the nucleation threshold fluence for water; the peak temperature is achieved at $16 \mathrm{~ns}$ after the onset of the excimer-laser irradiation and amounts to $426 \mathrm{~K}$. The peak temperatures for the test liquids obtained from the computation results are listed in Table 1, together with the boiling points of the liquids from the literature and the experimentally observed threshold fluences for nucleation, for which the computations were performed. Another important result of the calculations, as already discussed in Sect. 2.3 , is that the thickness of the superheated liquid layer is limited to a few hundred nanometers, as expected because of the low thermal conductivity of water.

\section{Conclusion}

In conclusion, we have demonstrated that the relatively simple optical reflectance probe is a powerful method for the study of nucleation dynamics at a liquid/solid interface in the nanosecond time scale, where the liquid film is superheated via pulsed-laser heating of the contacting solid surface. The excimer-laser threshold fluences for the nucleation of different liquids and liquid mixtures at the surface of a chromium film are determined. In addition the bubble-growth velocity for various test liquids is estimated to be of the order of $\mathrm{m} / \mathrm{s}$. Compared to other probe methods it is shown that the optical reflectance technique has a higher sensitivity in detecting the nucleation threshold and has further advantages of the applicability to opaque solid samples of any thickness. The threshold fluence for nucleation could also be determined by piezoelectric transducer measurements in a similar way.

Acknowledgements. The authors thank I. K. Pour, D. Krajnovich, and F. Lee of IBM Almaden Research Center. P. T. Leung of the Porland State Universiry, W. Zapka of IBM GMTC, Germany, and J. Boneberg of the University of Konstanz, Germany, for their valuable suppor, assistance, and contributions to this work. The help of X. Xu of the Universiry of Califomia, Berkeley, and M. Rubin and R. Furler of the Lawrence Berkeley Laboratories for the ellipsometry measurement is gratefully acknowledged. O. Yavas would like to acknowledge the financial suppon from the Friedrich-Ebert-Stiftung, Bonn, Germany. This work was supported in part by the Computer Mechanics Laboratory of the Universiry of Califomia at Berkeley.

\section{References}

1. V.P. Skripov, E.N. Sinitsyn, P.A. Pavlov, G.V. Ermakkov, G.N. Muratov, N.V. Bulanov. V.G. Baidakov: Thermophysical Propertit's of Liquids in the Melastable State (Gordon. Breach. New York 1988)

V.P. Skripov: Metasrable Liquids (Wiley, New York 1974)

2. J.G. Eberhart: J, Colloid Interface Sci. 56(2). 262 (1976)

3. C.T. Avedisian: J. Phys. Chem. Ref. Data 14(3), 695 (1985)

4. Y. Kagan: Russ. J. Phys. Chem. 34(1), 42 (1960)

5. H. Wakeshima, K. Takata: J. Phys. Soc. Jpn. 13, 1398 (1958) G.R. Moore: Am. Inst. Chem. Eng. J. 5, 458 (1959)

6. F.V. Bunkin, M.I. Tribel'skil: Sov. Phys. Usp. 30(2), 106 (1980)

7. D.R. Alexander, J.G. Armstrong: Appl. Opt. 26(3), 533 (1987) R.L. Armstrong: J. Appl. Phys. 56(7). 2142 (1984) 
8. B.S. Park, A. Biswas, R.L. Armstrong, R.G. Pinnick: Opt. Lett. 15(4), 206 (1990)

R.G. Pinnick, A. Biswas, R.L. Armstrong, S.G. Jennings, D. Pendleton, G. Femandez: Appl. Opt. 29(7), 918 (1990)

9. A.C. Tam, W.P. Leung, W.P. Zapka, W. Ziemlich: J. Appl. Phys. 71(7), 3515 (1992)

10. W. Zapka, W. Ziemlich, A.C. Tam: Appl. Phys. Let. 58, 2217 (1991)

11. W.P. Leung, A.C. Tam: Appl. Phys. Lett. 60(1), 23 (1992)

12. P.T. Leung, N. Do, L. KJees, W.P. Leung, F. Tong, L. Lam, W. Zapka, A.C. Tam: J. Appl. Phys. 72(6), 2256 (1992)

13. H.K. Park, X. Xu, C.P. Grigoropoulos, N. Do, L. Klees, P.T. Leung, A.C. Tam: Appl. Phys. Lett. 61(7), 749 (1992)

14. A.C. Tam, N. Do, L. Klees, P.T. Leung, W.P. Leung: Opt. Lett. 24(17), 1809 (1992)

15. O. Yavas, P. Leiderer, H.K. Park, C.P. Grigoropoulos, C.C. Poon, W.P. Leung, N. Do, A.C. Tam: Phys. Rev. Lett. 70(12), 1830 (1993)

16. J.C. Maxwell-Gamett: Philos. Trans. R. Soc. London 203 (1904)

17. A.P. Cracknell: Ultrasonics (Whykeham, London 1980)

18. H.K. Park, C.P. Grigoropoulos, O. Yavas, W.P. Leung, C.C. Poon, A.C. Tam: In Proc. 29th ASME Nat'l Heat Transfer Conference, Atlanta, GA, Vol. HTD 253 (American Society of Mechanical Engineers, New York 1993) p. 95

19. J.B. Watchman, Jr., W.E. Tefft, D.G. Lam, R.P. Stinchfield: J. Res. Natl. Bu. Stand., Sect. A 64(3), 213 (1960)

20. D.H. Trevena: Covitation and Tension in Liquids (Adam Hilger, Bristol 1987)

21. J. Bardina: In Particles on Surfaces, ed. by K.L. Mittal. Vol. 1 (Plenum, New York 1988) p. 327
22. J. Bodensohn, S. Klesy, P. Leiderer: Europhys. Lett. 8, 59 (1989)

23. V.P. Carey: Liquid-Vapor Phase-Change Phenomena (Hemisphere, Washington, 1992)

24. H.C. van de Hulst: Light Scattering by Small Particles (Dover, New York 1981)

25. M. Bom, E. Wolf: Principles of Optics (Pergamon, Oxford 1991)

26. R.C. Weast (ed.): CRC Handbook of Chemismy and Physics, 68th edn. (CRC, Boca Raton 1987)

27. A. Prospereni, M.S. Plesset: J. Fluid Mech. 85(2), 349 (1978)

28. S. Fujikawa, T. Akamatsu: J. Fluid Mech. 91, 481 (1980)

29. J.E. Shepherd, B. Sturtevant: J. Fluid Mech. 121, 379 (1982)

30. L.E. Nielsen: Predicting the Properties of Mixtures (Dekker, New York 1978)

31. A.C. Tam: Rev. Mod. Phys, 58, 381 (1986)

32. A. Polman, W.C. Sinke, M.J. Uttormark, M.O. Thomson: J. Mater. Res. 4(4), 843 (1989)

33. I. Thormahlen, J. Straub, U. Grigull: J. Phys. Chem. Ref. Data 14(4), 933 (1985)

34. K. Vedam, P. Limsuwan: Phys. Rev. Lett. 35(15), 1014 (1975)

35. N.F. Bunkin, V.B. Karpov: JETP Lett. 52, 18 (1990)

N.F. Bunkin, F.V. Bunkin: Sov. Phys. JETP 74(2), 271 (1992)

36. M.N. Ozisik: Boundary Value Problems of Heat Conduction (Dover, New York 1989)

37. Y.S. Touloukian: Thermophysical Properties of Maner, Thermal Conductivity (Plenum, New York 1970)

38. G. Henderson, C. Weaver: J. Opt. Soc. Am. 56(11), 1551 (1966)

39. E.D. Palik (ed.): Handbook of Oprical Constants of Solids I (Academic, New York 1985)

40. A.P. Prishivalko, S.T. Leiko: J. Appl. Spectrosc. 33, 1137 (1980)

41. F. Franks (ed.): Water (Plenum, New York 1972) 